\title{
ЭФФЕКТИВНОСТЬ ПРОИЗВОДСТВЕННО-СБЫТОВЫХ СИСТЕМ НОВОГО ПОКОЛЕНИЯ *
}

\author{
(c) 2019 Систер Владимир Григорьевич \\ доктор технических наук, профессор, чл.-кор. РАН \\ генеральный директор ООО «Национальная инновационная компания», Россия, Москва
}

(c) 2019 Горлачева Евгения Николаевна

кандидат экономических наук, доцент

МГТУ им. Н.Э. Баумана, Россия, Москва

E-mail: gorlacheva@yandex.ru

(c) 2019 Василенко Анна Павловна

ведущий специалист ООО «Национальная инновационная компания», Россия, Москва

Исследуется проблема обеспечения эффективности производственно-сбытовых систем нового поколения. Показано, что производственно-сбытовые системы нового поколения должны соответствовать современным требованиям по производительности, соотношению достигаемого результата к затратам в условиях технологической неопределенности. В работе предложен логистический подход к созданию производственно-сбытовых систем нового поколения, позволяющего использовать методы структурного проектирования для обеспечения эффективности подобных систем.

Ключевые слова: производственно-сбытовые системы нового поколения, цифровизация, логистический подход, структурное проектирование, технологическая неопределенность, индустрия 4.0, конвергенция, синхронизация.

\section{Введение}

Развитие ИТ-технологий и трансформация экономического пространства, породившие феномен «цифровой экономики» обуславливают изменения бизнес-среды производственно-сбытовых систем и предъявляют новые требования к обеспечению их эффективности [9]. Можно выделить ряд задач, стоящих перед производственно-сбытовыми системами (ПСС):

- встраивание в индустрию 4.0, переход к гибким производственным системам, обеспечение адаптивной производственной инфраструктуры;

- рациональное повышение роста затрат на НИОКР, обеспечивающих реализацию научно-технической политики непосредственно в процессе хозяйственной деятельности;

- неопределенность экономической конъюнктуры.

Производственно-сбытовые системы реагируют на изменения с разной скоростью и разными затратами. Однако любая производственно-сбытовая система заинтересована в своей конкурентоспособности в долгосрочной перспективе. Происходящие изменения приводят к тому, что для ПСС нового поколения характерны следующие специфические характеристики:

- интеграция в рамках производственных сетей информации и данных, отражающих все аспекты требований конструирования, проектирования;

- существенный научно-технический задел;

- глобализация команды разработчиков;

- формирование производственной «экосистемы» за счет кооперации и снижения барьеров между разными уровнями поставщиков узлов и комплектующих.

В этих условиях управление эффективностью ПСС становится нетривиальной задачей. Меняется само понятие эффективности. Если в традиционной трактовке под эффективностью понималось соотношение результата к затратам, то в современном понимании эффективность соответствие меняющимся требованиям за счет совокупности воздействий на структурно-функциональное развитие производственно-сбытовых систем, приводящих к оптимальным затратам на единицу произведенной продукции. Достижение заданного уровня эффективности возможно за счет учета этих требований при

* Исследование выполнено в рамках гранта РФФИ №19-010-00348\19 
проектировании производственно-сбытовых систем. Разрабатываемый логистический подход к управлению эффективностью ПСС нового поколения, позволяет использовать методы структурного проектирования для обеспечения эффективности.

Статья структурирована следующим образом: в первой части проанализированы вывозы научно-технологического развития на современном этапе; во второй части исследованы теоретико-методологические аспекты категории эффективности; в третьей части определены особенности ПСС нового поколения, в четвертой части разработан логистический подход к проектированию ПСС; в пятой части представлены выводы и заключение.

Вызовы научно-технологического развития на современном этапе

Основной проблемой современных ПСС является инновационная неопределенность, которую можно описать как меру незнания потенциальных возможностей научно-технологического развития. Развитие системы по S-образной кривой приводит рано или поздно к насыщению, и затраты на модернизацию системы становятся бесполезными [4]. Следующий виток развития возможен при переходе системы на новые принципы действия, т.е. преодоление технологического разрыва. Под технологическим разрывом в развитии производственно-сбытовой системы понимают качественный скачок в динамике целевых характеристик системы, разрыв ее эволюционного развития. Особое значение управление эффективностью ПСС приобретает в связи с проблемой сингулярности, иллюстрируемой «кривой Панова-Снукса», свидетельствующая о приближении границы инновационной неопределенности к длительности этапов НИОКР [3]. Уменьшение инновационной неопределенности ограничено существующим и перспективным технологическим укладом в экономике и общими закономерностями развития. Как отмечают в работе [2], необходимо учитывать взаимовлияние потенциальных противоречий во всех средах социума, что соответствует представлениям о седьмом технологическом укладе [6]. Основной характеристикой которого является превращение человеческого сознания в производительную силу (сознательное управление реальностью) на основе конвергенции нано-, био-, информационных технологий. В этой связи управление эффективностью должно быть непрерывным и опережающим по отношению к другим процессам, реализуемым на уровне ПСС.

теоретические проблемы управления эффективностью

Проблема управления эффективностью с определением оптимальных параметров ее функционирования и устойчивого развития одна из классических [1]. Сложность понятия эффективности обусловлена отсутствием единой интерпретации этого феномена.

Представленное исследование феномена «эффективности» [7] позволяет прийти к выводу, что эффективность является многоаспектным понятием и рассматривается как отношение результата к затратам, как степень фактического и ожидаемого результата желаемому; как степень достижения цели.

Клейнер Г.Б. [1] выделяет следующие категории эффективности:

целевая - уровень качества работы исследуемой системы ее целевому назначению;

технологическая или ресурсная - рациональность использования ресурсов предприятия;

экономическая - полезность результата деятельности с учетом платежеспособного спроса; внутренняя - оценка результативности;

внешняя - степень удовлетворения общественных потребностей предприятием;

статическая - краткосрочная оценка деятельности предприятия;

динамическая - стратегическая оценка деятельности предприятия в условиях инновационной неопределенности.

Рассмотренные категории эффективности отражают лишь отдельные аспекты и не рассматривают структурно-функциональные взаимосвязи в производственно-сбытовых системах.

Производственно-сбытовые системы характеризуются иерархичностью управления, активностью отдельных подсистем и сложной структурой; функционируют в виде взаимной и кумулятивной обусловленности подсистем в рамках своей структуры и определяются устоявшимися связями, сложившимися в конкретных социальных, экономических, политических, правовых и институциональных условиях.

Исходя из этого, управление эффективностью ПСС можно определить как целенаправленную совокупность мероприятий, влияющих на условия устойчивого развития множества ее элементов и расширения спектра инвариантных 
взаимосвязей между ними с целью обеспечения процесса воспроизводства в динамичном контексте. Что, в свою очередь, требует разработки логистического подхода к проектированию ПСС нового поколения.

\section{Особенности ПСС нового поколения}

Современные промышленные предприятия работают в сложных условиях, которые характеризуются перенасыщением рынков, резкими изменениями в динамике рыночного спроса, возрастающим разнообразием продукции и уменьшающимися объемами партий выпускаемых изделий. В таких условиях вопросы обеспечения эффективности становятся нетривиальной задачей. Повышаются требования к стратегическому планированию и учету требований эффективности на этапе проектирования. Согласно Корену [8], ПСС нового поколения должны соответствовать следующим требованиям:

- обладать возможностями для быстрого реагирования;

- постоянно улучшать качество выпускаемых изделий;

- минимизировать затраты на производство.

С одной стороны, такие требования можно выполнить в условиях высокой стабильности и отлаженных производственных процессов. С другой стороны, эти требования могут быть выполнены за счет своевременного реагирования на отклонения производственной системы, производственного процесса или конфигурации изделий в соответствии с общими целевыми производственными показателями. Однако эффективность таких адаптивных и быстро реагирующих производственных систем зависит от синхронизированной с производственным процессом информации, поступающей в режиме реального времени от производственной системы, производственного процесса и отдельного изделия.

Использование современных информационно-коммуникационных технологий позволяет собирать оперативную информацию с ПСС. Этот процесс можно рассматривать как качественный переход от систем управления, работающих офлайн, к системам управления, работающих онлайн.

Традиционная автоматизация не способна справиться с возрастающей сложностью современных ПСС. Необходимы интеллектуальные системы, позволяющие реагировать на непредсказуемые ситуации, планировать свои дальнейшие действия, способные к обучению (машинное обучение) [8].

ПСС нового поколения оборудованы искусственными датчиками и механизмами управления, интегрированные и встроенные в физические системы. Их отличие в реализации рефлексивного поведения, происходит за счет использования методов и моделей «машинного обучения». Пример архитектуры ПСС нового поколения приведен на рис. 1.

ПСС нового поколения способны «обучаться» на опыте ранее осуществленных задач и поэтому адаптируются к параметрам новых изделий, производя мелкосерийные и индивидуальные

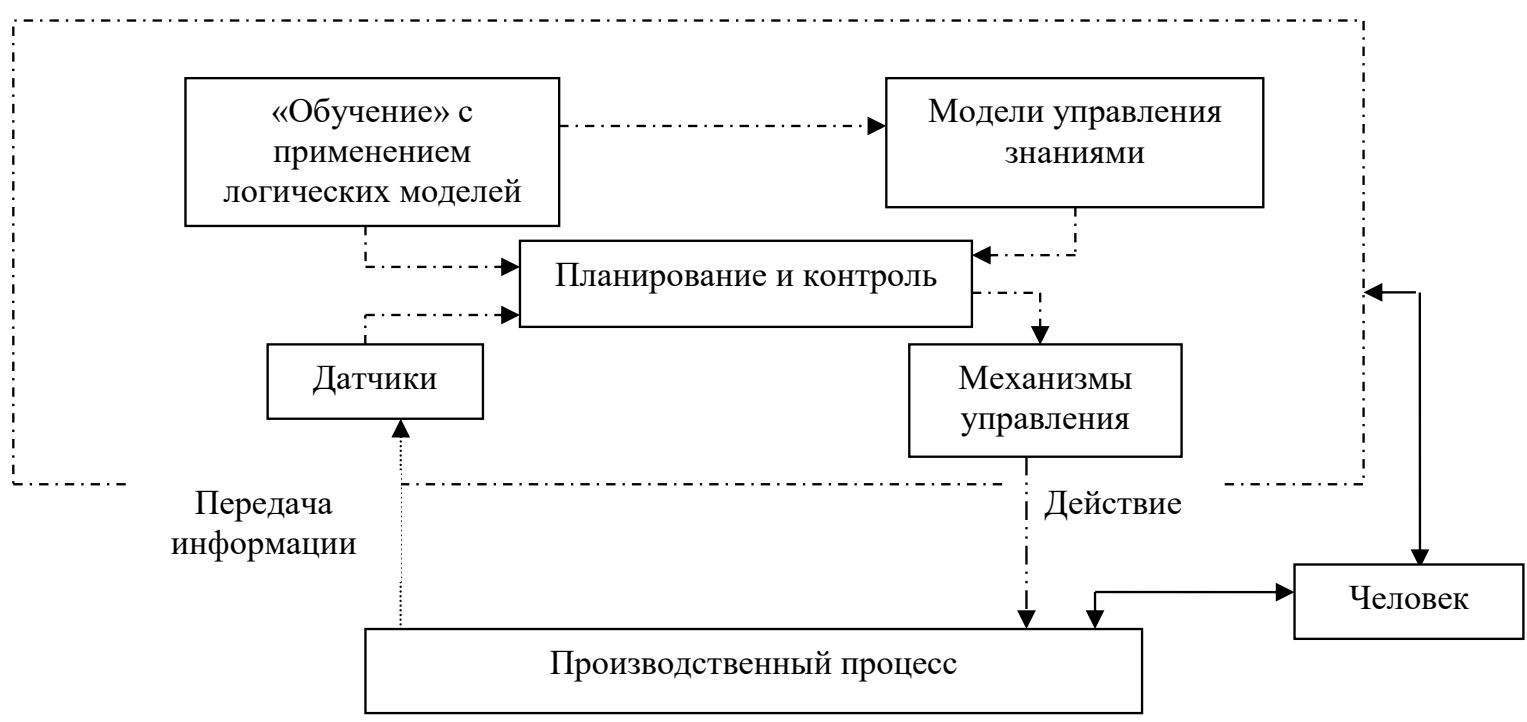

Pис. 1. Пример ПСС нового поколения 
заказы по конкурентоспособным ценам. ПСС нового поколения сочетают преимущества автоматизированных систем (низкие издержки, высокое качество, высокая эффективность и небольшие сроки производства) с гибкостью, адаптивностью обычных цехов, не оснащенных системами автоматизации, реализуя, таким образом, процессно-ориентированное управление (рис. 2).

Приведем примеры типовых структур ПСС нового поколения [5]:

Базовая ПСС: платежеспособный спрос определяет выпускаемый продукт, заданы все виды затрат, ПСС проектируется с минимальным количеством элементов, инвестициями и расходами по эксплуатации;

ПСС с высоким уровнем производительности: реализует выполнение требований радикального сокращения сроков поставки;

Высокотехнологичная ПСС: проектируется для выпуска продукции, требующей масштабны инноваций, при этом разрыв по времени между разработкой и сроком внедрения должен быть минимальным;

Интегрируемая ПСС: изначально проектируется с соответствующим уровнем вертикальной интеграции (не ниже 20\%), предполагает, что производственный цикл не превышает семи часов; на первый план выходит кардинальное снижение сложности в цепи создания стоимости и интеграция с системными поставщиками;

Адаптивная ПСС: конкурентоспособность зависит от адаптации к изменениям, а также от возможностей оперативно и экономически рационально проводить необходимые организационные процессы.

Представленная типология свидетельствует о том, что ПСС нового поколения отличаются от традиционных в следующих аспектах:

- на уровне продукта (концепция платформы, разработка продукции с учетом особенностей производства и монтажа);

- на уровне производства (интегрированные косвенные функции, сценарии автоматизации);

- на уровне логистики (отношения с внутренними поставщиками, создание продукта на основе учетом логистических факторов, гибкие системы управления логистикой);

- на уровне организации (процесс организации виртуальных предприятий, междисциплинарные группы, предприятия-платформы).

Изменения в ПСС находят отражения и в стратегиях управления производством (табл. 1).

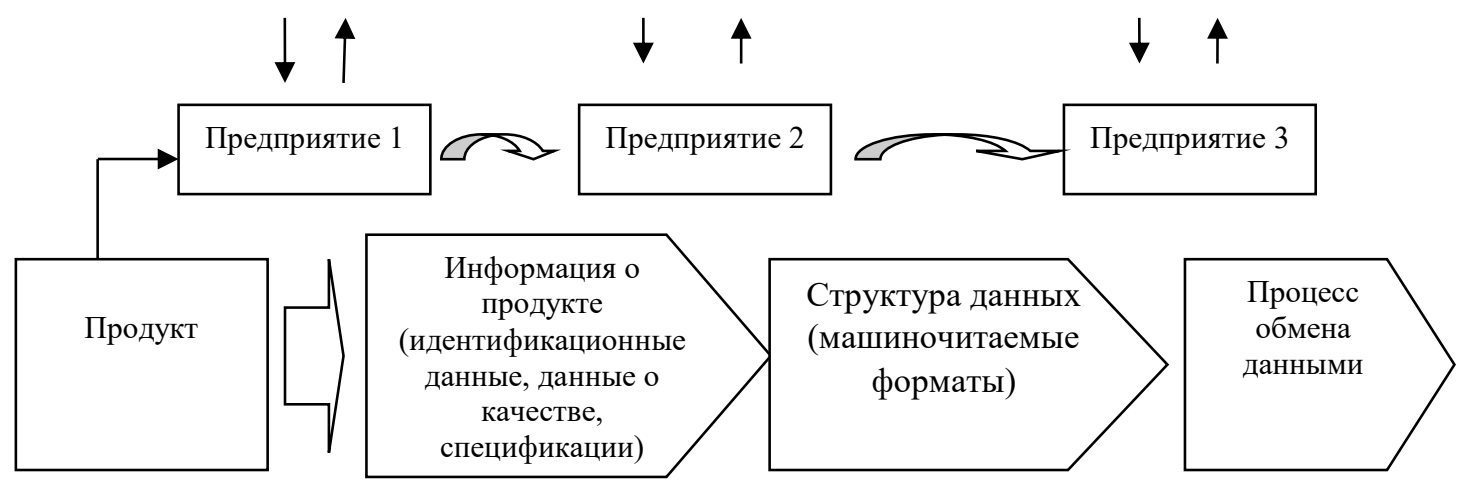

Puc. 2. Схематичное изображение процессно-ориентированного управления в ПСС нового поколения

Таблица 1. Различия в стратегиях управления производством в традиционных и новых ПСС

\begin{tabular}{|l|l|}
\hline Традиционные ПСС & ПСС нового поколения \\
\hline Ориентация на мощности & Ориентация на потоки информации \\
\hline Ориентация на длительность & Ориентация на продукт \\
\hline Выполнение программы & Выполнение под заказ \\
\hline Цикличная работа & Непрерывная работа \\
\hline Максимальное разделение труда & Минимальное разделение труда \\
\hline $\begin{array}{l}\text { Ориентация на экономически целесообразный объем } \\
\text { партии }\end{array}$ & Ориентация на штучное производство \\
\hline
\end{tabular}


Таким образом, управление эффективностью ПСС все больше зависит от того, в какой мере она соответствует постоянным изменениям динамического контекста окружающей среды. Что обуславливает необходимость разработки логистического подхода, позволяющего проектировать гибкие, с определенным запасом прочности, параметры эффективности.

Логистический подход к проектированию ПСС нового поколения

Разрабатываемый логистический подход к проектированию гибких параметров эффективности реализуется в трех аспектах: стратегическом, структурном, системном (рис. 3).

Эти аспекты характеризуют ресурсы, остающиеся постоянными на протяжении всего жизненного цикла выпускаемых продуктов. В этих условиях проектирование ПСС также включает учет, оптимизацию структуры продукции, распределение функций создания стоимости в глобальных производственных цепочках, процессы выпуска продукции, потоки материалов, а также вопросы снабжения и утилизации отходов.

На уровне стратегического аспекта форму- лируется система целей в ее динамике и направленности. Приоритетное значение имеют содержание и характеристика целей: выявляется их взаимозависимость, описывается иерархия и определяются потенциальные конфликты целей. Уровень их детализации зависит от характера проблемы и этапа планирования; также формулируются требования к рабочим системам (табл. 2).

Общая цель стратегического аспекта - обеспечить конкурентоспособность в долгосрочной перспективе.

На уровне структурного аспекта формируются релевантные системы; определяется структура продукта, основные процессные цепочки, необходимые ресурсы, формулируется проект идеальной системы.

На уровне системного аспекта исследуются вопросы экономической целесообразности, соответствие условиям поставленной задачи; анализируются системы изготовления продукции и монтажа, потоков материалов, складского хранения и транспорта, организационных систем с учетом их взаимозависимости. Несмотря на то, что системный аспект идентичен со структур-

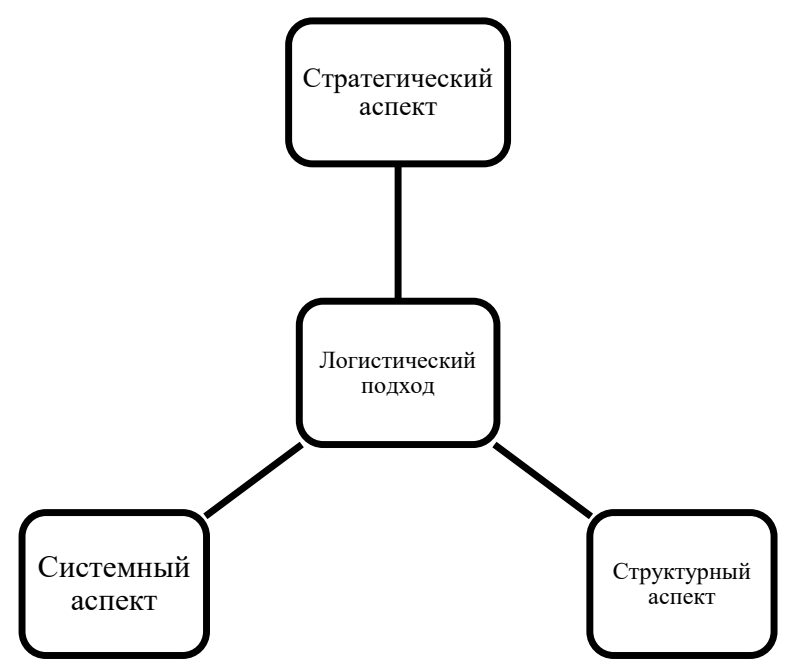

Puc. 3. Аспекты разрабатываемого логистического подхода

Таблица 2. Требования к рабочим системам

\begin{tabular}{|l|l|}
\hline Рабочие системы & Факторы, влияющие на параметры эффективности \\
\hline Продукт & Ориентация на заказы \\
\hline Технология & $\begin{array}{l}\text { Объединение ресурсов позволяют повысить уровень автоматизации; } \\
\text { высокие затраты на технологии обуславливают необходимость в } \\
\text { высокой производительности на единицу площади }\end{array}$ \\
\hline Организация & $\begin{array}{l}\text { Используются механизмы координации, контуры автоматического } \\
\text { регулирования, механизмы самоорганизации }\end{array}$ \\
\hline Оборудование & Должно обеспечивать гибкость и высокую производительность \\
\hline
\end{tabular}


ным, по основным стадиям реализации, их различие заключается в степени детализации. Так, структурный аспект включает:

- область анализа - производственно-сбытовая система;

- подсистему «функциональная система» (участок изготовления, склад);

Системный аспект включает:

- область анализа - функциональная система;

- подсистему «рабочий сегмент».

На рис.4 представлена подробная схема реализации логистического подхода.

ПСС нового поколения должны ежедневно реагировать на запросы рынка, что требует гармоничного взаимодействия производства и логистики.

Серьезной проблемой в управлении эффективностью ПСС является то, что в недостаточной мере учитывается текущая или требуемая организация производства, а также соответствующие информационные потоки.

В ПСС нового поколения система пунктов управления встраивается как координирующий модуль, который обеспечивает сопряжение с системой управления производством и ресурсами и распределение данных между отдельными пунктами управления. Адаптивные

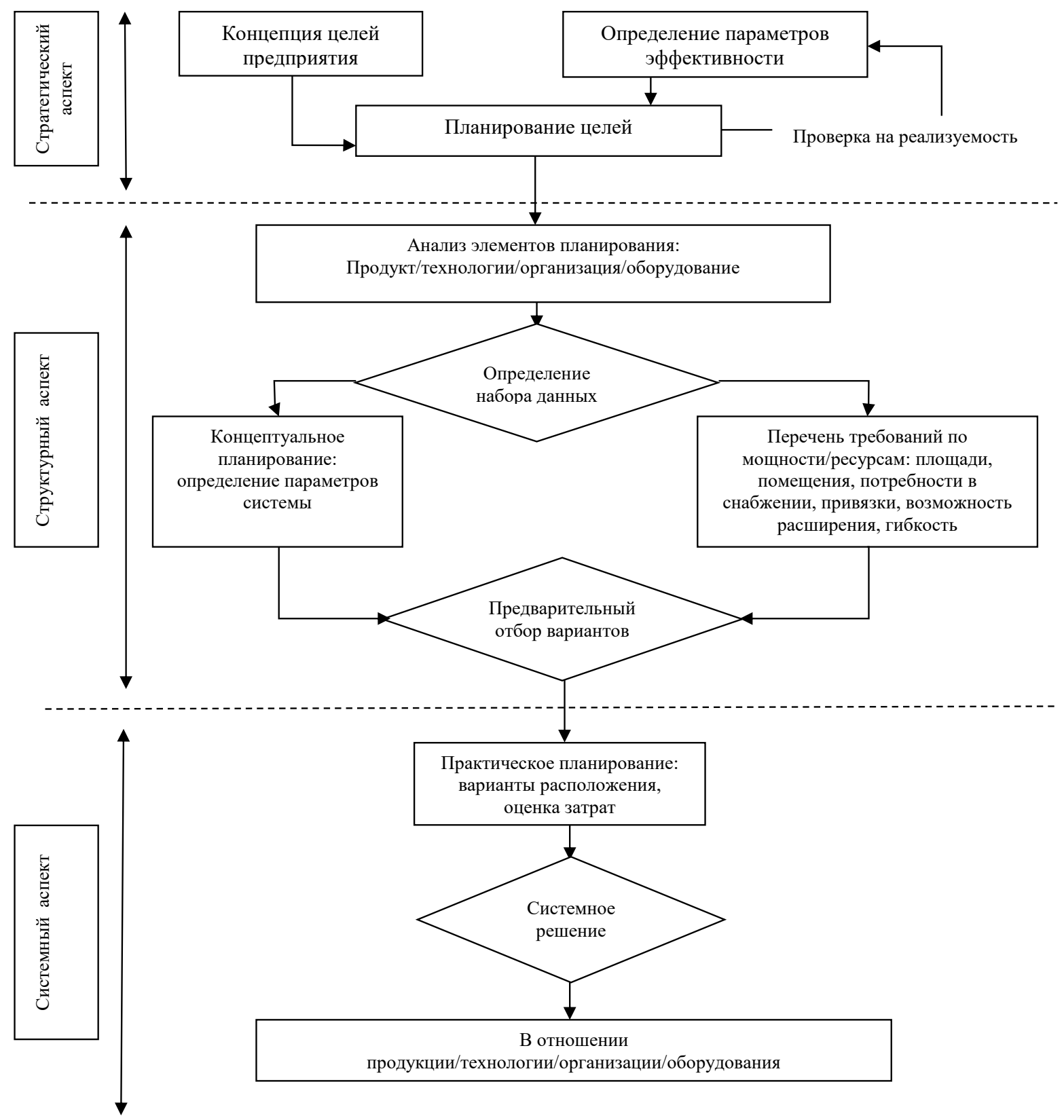

Puc. 4. Реализация логистического подхода в управлении эффективностью ПСС нового поколения 
пункты управления основываются на логистике предприятия. ПСС делится на элементы по горизонтали - по ходу потока материалов - и по вертикали в соответствии с уровнями принятия решений и выполнения работ:

- при горизонтальном объединении отдельные области структурируются по ходу потока материалов;

- при вертикальном объединении предприятия характеризуется уровнями принятия решений и выполнения работ:

- уровень потока материалов отражает самый нижний уровень - физический производственный процесс;

- уровень логистики связан с потоком материалов через поток информации. На логистическом уровне сводятся воедино все сведения, накапливаемые по ходу потока материалов;

- уровень управления является самым высоким. Обобщенная информация, отфильтрованная на логистическом уровне, используется для стратегического планирования.

Логистический подход в управлении эффективностью реализуется через:

- параллельное применение нескольких равноправных концепций управления;

- децентрализованное управление и распределение функций и задач;

- программная поддержка одинаковой приоритетности на всех уровнях планирования;

- формирование контуров управления;

- одновременное планирование ресурсов;

- создание единой модели данных для планирования и управления, отражающей процессы потока материалов с их зависимостями.

За счет предложенного логистического подхода ПСС нового поколения достигает заданных параметров эффективности: точное соблюдение сроков в условиях коротких жизненных циклов, снижения уровня запасов за счет интеграции снабженческих процессов. Логистический подход позволяет организовать структурные взаимосвязи между ресурсами, согласовать их друг с другом, позволяя ПСС достигать эффективности на новом качественном уровне.

Заключение

Рыночные трансформации и изменение технологий происходят все интенсивнее, что требует более быстрой адаптации производственно-сбытовых систем. Ряд авторов считает, что идея цифровой производственно-сбытовой системы станет ведущей в ближайшие годы. В это понятие включают широкий набор цифровых моделей, методик и инструментов моделирования производственных процессов. В компьютерной системе, образующей ядро «цифровой производственно-сбытовой системы» реализуется комплексная модель продукции, процессов и ресурсов, позволяющая всем задействованным в производственном процессе лучше представлять ситуацию в целом. Современные программные решения устанавливают непосредственные связи между всеми аспектами хозяйственной деятельности, реализуя «синхронное конструирование» от первых эскизов и планирования до запуска в производство.

Основными характеристиками стратегии цифрового производства являются:

- тесное сопряжение НИОКР и планирования;

- заблаговременное изучение критических объемов и монтажных циклов;

- оперативный обмен информацией между отделами;

- поддержка и ускорение производственной деятельности.

Предложенный логистический подход позволяет интегрировать различные аспекты деятельности в комплексное развитие производственно-сбытовых систем нового поколения. Реализация управления эффективностью происходит за счет текущей интеграции меняющихся базовых условий и постоянной адаптации ПСС к ним.

Исследование выполнено в рамках гранта РФФИ № 19-010-00348 19.

\section{Библиографический список}

1. Клейнер Г.Б., Рыбачук М.А. Системная сбалансированность экономики. М.: Из-во «Научная библиотека». 2017-320 c.

2. Клюшников В.Ю., Романов А.А., Тюлин А.Е. Методология создания инновационного научно-технического задела в ракетно-космической отрасли // Ракетно-космическое приборостроение и информационные системы, 2018. Том 5, выпуск 2, С.53-64. 
3. Клюшников В.Ю. Методология комплексного прогнозирования технологического развития ракетно-космической техники // Космонавтика и ракетостроение, 2017, № 2(95), С.13-25.

4. Нижегородиев Р. М. Логистическое моделирование экономической динамики (ч.1) // Проблемы управления. 2004. С.46-53.

5. Павеллек Г. Комплексное планирование промышленных предприятий: базовые принципы, методика, ИТ-обеспечение. М.: Альпина Паблишер, 2015. 366 с.

6. Прохоров И.А. Начало 7-го технологического уклада. Режим доступа: www.energoforum.org/pointofview/ prohorov/7-tech-structure.aspx (дата обращения: 27.03.2019)

7. Смирнов A.А. Исследование проблемы организации теоретической и практической деятельности по повышению эффективности социально-экономического развития региона // Аудит и финансовый анализ. 2010. № 3 C.0-12. Режим доступа: http://auditfin.com/fin/2010/3/10_06.pdf (дата обращения: 23.03.2019).

8. Koren Y. General RMS characteristics: comparison with dedicated and flexible systems // Reconfigurable manufacturing systems and transformable factories, A.I. Daschenko, ed. Berlin, Germany: Springer-Verlag, 2006, pp.27-45.

9. Omelchenko I.N., Drogovoz P.A., Shiboldenkov V.A., Gorlacheva E.N., Yusufova O.M. The modelling of the efficiency in the new generation manufacturing distributive systems based on the cognitive production factors / Proceedings of the 3rd International Conference on Mechanical, System and Control Engineering (ICMSC2019), in press 